\title{
Adaptive Cell Sectorization for CDMA Systems
}

\author{
Cem U. Saraydar and Aylin Yener
}

\begin{abstract}
Given the user distribution in a cell, we investigate the two problems of how to appropriately sectorize the cell such that we minimize the total received power and the total transmit power of all the users, while giving each user acceptable quality of service in both cases. For the received power optimization problem, we show that the optimum arrangement equalizes the number of users in each sector. The transmit power optimization is formulated as a graph partitioning problem that is polynomially solvable. We provide an algorithm that finds the best sectorization assignment as well as the optimal transmit powers for all the users. The computational complexity of the algorithm is polynomial in the number of users and sectors. For both the received power optimization and the transmit power optimization, under nonuniform traffic conditions, we show that the optimum arrangement can be quite different from uniform cell sectorization (equal width sectors). We also formulate and solve the transmit power optimization and cell sectorization problem in a multicell scenario that would improve the capacity of a hot spot in the network. We observe that, with adaptive sectorization, where the sector boundaries are determined in response to users' locations, received and transmit power savings are achieved, and the number of users served by the system (system capacity) is increased compared to uniform sectorization of the cell.
\end{abstract}

Index Terms-Adaptive sectorization, CDMA, graph partitioning, interference management, power control, resource management.

\section{INTRODUCTION}

D UE TO THE ever-increasing demand for wireless services, efficient use of resources to maximize capacity remains of utmost importance for system design. Among the ambitious goals of the wireless systems are high quality service and coverage for maximum number of subscribers. CDMA technology has emerged as a promising candidate to fulfill these goals [1].

It is well known that CDMA systems are interference limited, and interference management techniques are necessary to enhance the capacity of a CDMA system. Transmit power control, which minimizes the interference that users create for each other, is one important technique that has been studied in detail. Originally, the transmit power control problem was posed as an eigenvalue problem for nonnegative matrices, and the solution was found in a centralized and noniterative fashion [2], [3]. This was followed by the development of iterative and distributed algorithms that require only local measurements [4]-[6]. All these works assumed fixed resource assignment and one antenna per

Manuscript received September 2, 2000; revised November 1, 2000.

C. U. Saraydar was with Wireless Information Network Laboratory (WINLAB) Rutgers University. He is now with Bell Labs Advanced Technologies, Lucent Technologies, Holmdel, NJ 07733 USA.

A. Yener was with Wireless Information Network Laboratory (WINLAB) Rutgers University. She is now with Lehigh University, Bethlehem, PA 18015-3084 USA.

Publisher Item Identifier S 0733-8716(01)01910-2. base station. Joint transmit power control and base station assignment problem was studied in [7] and [8]. More recently, algorithms that consider joint transmit power control and receiver processing, either in the temporal domain via multiuser detection [9], or in the spatial domain via receiver beamforming with base station antenna arrays [10], or in both temporal and spatial domains [11], have been proposed and are shown to achieve capacity gains by further suppressing the interference compared to traditional power control. Other references related to the capacity analysis of CDMA systems with power control and receiver processing include [12] and [13]. A framework that identifies the convergence properties for iterative power control algorithms in the uplink is given in [6].

Another way of reducing the interference between users is to sectorize the cells using directional antennas. While this approach still utilizes the spatial domain to introduce orthogonalization to the system, it is fundamentally different than beamforming [14], [15]. Beamforming combines the received signals from multiple antennas in a unique way for each user to suppress the interference that user sees. Sectorization merely employs directional antennas and each user's signal is received at only one of these antennas. Since only a subset of the users is received at each antenna, the interference that each user sees is less compared to a single antenna system. No spatial combiner is used. While this is a more rigid scheme than beamforming, the simplicity of the resulting receivers is appealing and sectorization can be quite beneficial especially for static systems, i.e., if the users do not have to handoff from sector to sector frequently.

It has been shown that sectorization increases the number of users admissible in a system, i.e., system capacity [16]. However, under highly nonuniform traffic loads, conventional sectorization which divides the cell into equal width sectors might fail to bring much capacity improvement. If information is available about the geographic distribution of the call traffic, it may be possible to design sectors such that maximum capacity gain can be achieved. Recently, fixed wireless systems, wireless local loops (WLL), are posed as an alternative to conventional local wired telephone service [17]. Contrary to mobile macrocellular systems, for fixed wireless systems (or systems with low mobility), users' whereabouts, and thus their uplink gains to the base station, do not change much with time and the information about the traffic load as a function of the position in the cell can be obtained at the base station. It is then possible to use this information to sectorize the cells such that the maximum coverage is achieved, i.e., the maximum number of subscribers get their required quality of service.

In this paper, we investigate the minimization of the total power in a cell. Given the number of sectors and terminal locations, we study the best way of sectorizing the cell such that all subscribers in the cell meet their quality of service requirements 


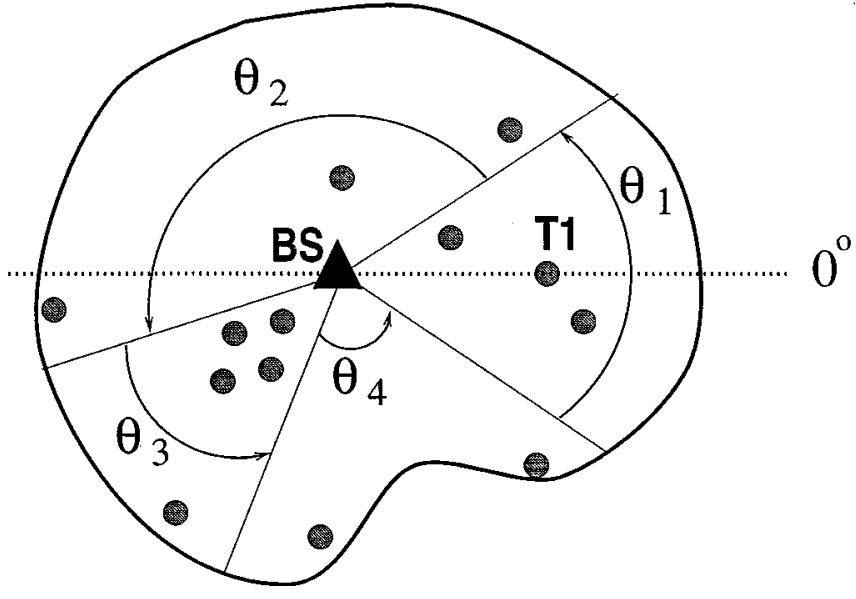

Fig. 1. Sectorization of a cell.

while using as little power as possible. We call this approach adaptive cell sectorization since the sector configurations are adaptive to the geographical load in the cell. The sector boundaries are designed in response to users' channel gains. We first consider a single cell system where we study the minimization of the total received power as well as the total transmit power. We show that in the received power optimization case, the best arrangement is such that the number of users in each sector is equalized (as much as possible) leading to a discrete waterfilling solution. The minimum total transmit power problem is formulated as a graph partitioning problem whose solution is found by solving a shortest path problem on a network of nodes constructed from the instance of the sectorization problem. We then formulate and solve the cell sectorization problem considering the effects of out-of-cell interference. We provide performance comparisons of solutions by both the received power and transmit power problems with the equal width sectors configuration (uniform sectorization) implemented in current systems. We observe that power savings are achieved and power assignments that are infeasible with uniform sectorization can be handled with nonuniform sectorization. Thus, more users can be supported by the cell.

\section{MODEL}

We first consider a single cell DS-CDMA system with processing gain $G$ and a total of $M$ users. The multiple cell scenario is studied in Section V. The user (call traffic) density in the cell is a known function of the distance and angle (referenced to the origin) from the base station. In a WLL, this information is readily available through the addresses of the subscribers. Note that the user density can be highly nonuniform, in which case partitioning the cell into equal width sectors as is done conventionally is not the best way of maximizing the number of users to be served by the cell. We assume the cell is to be partitioned into $N$ sectors and the antennas have ideal directivity, i.e., there is no interference between the sectors.

Our aim is to find the best sectorization arrangement such that all users get their required quality of service while expending as little power as possible. Fig. 1 shows an example of a sectorization configuration which is characterized by the reference point $\left(0^{\circ}\right.$ angle) and the vector containing sector width angles, $\theta$. In each sector, all users belonging to that sector interfere with each other. We assume that each user has a pseudorandom signature sequence and matched filters are employed at the base station. We consider a synchronous system for simplicity. The quality of service measure is the signal-to-interference ratio (SIR). A user has reliable communication if its SIR at its assigned sector antenna is larger than a common target SIR, $\gamma^{*}$.

\section{RECEIVED POWER OPTIMIZATION}

We first consider the optimization problem of minimizing total received power at the base station by choosing the transmit power for each user and a sector partition. Although the focus of this study is the transmit power minimization problem, we first analyze the received power minimization problem for reasons that will become clear by the end of this section.

We consider the problem of optimally placing $M$ terminals into $N$ sectors such that the total power received at the base station is minimized while ensuring each user maintains the target SIR of $\gamma^{*}$. Let $s_{i}(\boldsymbol{\theta})$ denote the set of users that reside in the area spanned by sector $i$, and $\boldsymbol{\theta}$ denote the $N$-tuple vector that contains the sector angles. Since sectorization creates disjoint subsets of the set of terminals, each terminal belongs to only one of the sets $s_{i}(\boldsymbol{\theta})$ where $i=1, \ldots, N$. We denote the sector index that user $j$ belongs to by $a_{j}$. The power expended by terminal $j$ is $p_{j}$ which is received after being attenuated by its channel gain $h_{j}$. The SIR of terminal $j$ at the output of its matched filter can be expressed as

$$
\gamma_{j}=\frac{p_{j} h_{j}}{\sum_{k \neq j} \delta_{k j} p_{k} h_{k} / G+\sigma^{2}}
$$

where $\sigma^{2}$ is due to AWGN and $\delta_{k j j}$ is defined as

$$
\delta_{k j}= \begin{cases}1, & \text { if } a_{j}=a_{k} \\ 0, & \text { otherwise. }\end{cases}
$$

Notice that each terminal experiences interference from the terminals in its own sector only. Although the $\delta_{k j}$ notation seems complicated, it will be convenient for the graph theoretic formulation in Section IV-B.

The received power optimization problem can be expressed as

$$
\begin{array}{ll}
\min _{\boldsymbol{\theta}, \mathbf{p}} & \sum_{i=1}^{N} \sum_{j \in s_{i}(\boldsymbol{\theta})} p_{j} h_{j} \quad(\mathbf{R P}) \\
\text { s.t. } & \gamma_{j}=\frac{p_{j} h_{j}}{\sum_{k \neq j} \delta_{k j} p_{k} h_{k} / G+\sigma^{2}} \geq \gamma^{*} \\
& j=1, \ldots, M \\
& \mathbf{p} \geq \mathbf{0} \quad \mathbf{1}^{T} \boldsymbol{\theta}=2 \pi
\end{array}
$$

where $\mathbf{0}$ and $\mathbf{1}$ denote the all zero and all 1 vectors, respectively. Note that the constraints represent each user's reliable connection requirement.

It is not hard to see that the SIR constraints ought to be satisfied with equality at the solution of (3) [6]. Note also that within each 
sector, minimum power is achieved by assigning equal received powers to all users belonging to the sector. Denote the received power in sector $i, q_{i}^{*}$, such that $q_{i}^{*}=p_{j} h_{j}$ for all users $j$ in sector $i$. Given the above observations, we can express $q_{i}^{*}$ as

$$
q_{i}^{*}=\frac{\gamma^{*} \sigma^{2}}{1-\frac{\gamma^{*}\left(M_{i}(\boldsymbol{\theta})-1\right)}{G}}
$$

where $M_{i}(\boldsymbol{\theta})=\left|s_{i}(\boldsymbol{\theta})\right|$ is the number of users in sector $i$, i.e., the cardinality of set $s_{i}(\boldsymbol{\theta})$. Given (4), we can rewrite (3) as

$$
\begin{aligned}
& \min _{\boldsymbol{\theta}, \mathbf{q}^{*}} \sum_{i=1}^{N} M_{i}(\boldsymbol{\theta}) q_{i}^{*} \\
& \text { s.t. } \quad q_{i}^{*}=\frac{\gamma^{*} \sigma^{2}}{1-\frac{\gamma^{*}\left(M_{i}(\boldsymbol{\theta})-1\right)}{G}} \quad i=1, \ldots, N \\
& \mathbf{q}^{*} \geq \mathbf{0} \quad \sum_{i=1}^{N} M_{i}(\boldsymbol{\theta})=M \quad \mathbf{1}^{T} \boldsymbol{\theta}=2 \pi
\end{aligned}
$$

where $\mathbf{q}^{*}$ is the $N$-vector that contains the received power values associated with each sector. Optimization problem (5) is equivalent to

$$
\begin{aligned}
\min _{\boldsymbol{\theta}} & G \sum_{i=1}^{N} \frac{\sigma^{2} \frac{\gamma^{*} M_{i}(\boldsymbol{\theta})}{G}}{1-\frac{\gamma^{*}\left(M_{i}(\boldsymbol{\theta})-1\right)}{G}} \\
& M_{i}(\boldsymbol{\theta})<G / \gamma^{*}+1 \quad i=1, \ldots, N \\
& \sum_{i=1}^{N} M_{i}(\boldsymbol{\theta})=M \quad \mathbf{1}^{T} \boldsymbol{\theta}=2 \pi .
\end{aligned}
$$

Substituting $M_{N}(\boldsymbol{\theta})=M-\sum_{i=1}^{N-1} M_{i}(\boldsymbol{\theta})$ and taking derivatives with respect to $M_{j}$ reveals that the optimum point has to satisfy

$$
\frac{1}{1-\frac{\gamma^{*}}{G}\left(M_{j}(\boldsymbol{\theta})-1\right)}=\frac{1}{1-\frac{\gamma^{*}}{G}\left(M-\sum_{i=1}^{N-1} M_{i}(\boldsymbol{\theta})-1\right)}
$$

for all $j=1, \ldots, N$. Note that we have treated the number of users in each sector as continuous variables which is a relaxation. Since the space of integers is a subset of the continuous solution space, finding a set of integer values that satisfy (7) for all $j$ guarantees that we achieve the solution of (6). If we assume $M=k N$, where $k$ is an integer, then (7) implies that the sectors should be arranged, i.e., $\boldsymbol{\theta}$ should be determined, such that

$$
M_{j}(\boldsymbol{\theta})=M / N \quad j=1, \ldots, N .
$$

Thus, to minimize the total power received at the base station while all users get their minimum required quality of service, the cell should be sectorized such that each sector has the same number of active users. Clearly, any set of $\boldsymbol{\theta}$ for which each sector covers $M / N$ users is optimum. So, starting from one reference point, the sectorization can be arranged such that each sector is terminated when a coverage of $M / N$ users is reached.

Note that the generalization of the result to $M / N$ not an integer is immediate in the sense that the left over users after $k=\lfloor M / N\rfloor$ are placed in each sector, should be distributed evenly to the sectors. Thus, as a result, some sectors have to serve $k+1$ users while some serve $k$. The total interference is placed as equally as possible which is the discrete water-filling solution.

One would consider that designing sector boundaries such that each sector contains an equal number of terminals is an intuitive sectorization method. In this section, we have shown that indeed such a method has an analytical basis and emerges as a solution to the received power minimization problem. We will use this result to compare results from sectorization by transmit power minimization.

\section{TRANSMIT POWER OPTIMIZATION}

\section{A. Problem Statement}

Using the notation introduced in Section III, the transmit power optimization problem can be stated in a similar fashion as

$$
\begin{array}{ll}
\min _{\boldsymbol{\theta}, \mathbf{p}} & \sum_{i=1}^{N} \sum_{j \in s_{i}(\boldsymbol{\theta})} p_{j} \quad(\mathbf{T P}) \\
\text { s.t. } & \gamma_{j}=\frac{p_{j} h_{j}}{\sum_{k \neq j} \delta_{k j} p_{k} h_{k} / G+\sigma^{2}} \geq \gamma^{*} \\
& j=1, \ldots, M \\
& \mathbf{p} \geq \mathbf{0} \quad \mathbf{1}^{T} \boldsymbol{\theta}=2 \pi .
\end{array}
$$

The observations that led to (4)-i.e., 1) SIR constraints are satisfied with equality at the optimum, and 2) within each sector, minimum power assignment is such that each user in that sector is received with the same power at the base station-also hold for (9). Thus, we can rewrite (9) as

$$
\begin{array}{lll}
\min _{\boldsymbol{\theta}, \mathbf{q}^{*}} & \sum_{i=1}^{N} q_{i}^{*} \sum_{j \in s_{i}(\boldsymbol{\theta})} \frac{1}{h_{j}} & \\
\text { s.t. } & q_{i}^{*}=\frac{\gamma^{*} \sigma^{2}}{1-\frac{\gamma^{*}\left(M_{i}(\boldsymbol{\theta})-1\right)}{G}} & i=1, \ldots, N \\
& \mathbf{q}^{*} \geq \mathbf{0} \quad \sum_{i=1}^{N} M_{i}(\boldsymbol{\theta})=M & \mathbf{1}^{T} \boldsymbol{\theta}=2 \pi
\end{array}
$$

which is equivalent to

$$
\begin{aligned}
\min _{\boldsymbol{\theta}, M_{i}} & \sum_{i=1}^{N} \frac{\gamma^{*} \sigma^{2}}{1-\frac{\gamma^{*}\left(M_{i}(\boldsymbol{\theta})-1\right)}{G}} \sum_{j \in s_{i}(\boldsymbol{\theta})} \frac{1}{h_{j}} \quad(\mathbf{T P}) \\
& M_{i}(\boldsymbol{\theta})<G / \gamma^{*}+1 \quad \sum_{i=1}^{N} M_{i}(\boldsymbol{\theta})=M \quad \mathbf{1}^{T} \boldsymbol{\theta}=2 \pi .
\end{aligned}
$$

It is worthwhile to note that the set of feasible solutions for RP given by (6) and the one for TP given by (11) are identical since the constraints of both problems are the same. This translates to the system supporting the same maximum number of people for RP and TP. Transmit power optimization brings to the system 
the additional advantage that the resulting transmit powers while supporting a certain number of people will be lower than the transmit power values emerging from the solution of RP, leading to longer battery life for each subscriber.

We should also note that the joint sector assignment and transmit power control problem is different from those that can be unified by the theory of standard interference functions described in [6]. In particular, although an iterative solution to the transmit power control problem under a specific sectorization arrangement is possible using the framework in [6], this is not the case where an optimization over the sectorization arrangements is added to the problem. In this case, the problem becomes similar to joint optimal power control and frequency/time slot assignment and is reminiscent of bin packing problems [18]. Fortunately, it is possible to formulate the joint sector assignment and transmit power control problem as a graph theoretic problem that is solvable in polynomial time. In particular, we observe that optimization problem (11) can be formulated as a graph partitioning (GP) problem. We also observe that because of the special topology of the problem at hand, this problem is polynomially solvable [19], [20]. We will first discuss how the physical wireless system entities can be represented in a graph setting. The equivalent graph theoretic problem is then defined and the solution is presented. We give an algorithm that solves the TP problem. We then discuss the constrained problem where sector width is upper and lower bounded and sector boundaries are not allowed between close terminals. Finally, sectorization in a multicell system is examined in Section V.

\section{B. Graph Theoretic Formulation of TP}

TP does not have a closed-form solution. However, through appropriate transformations, it can be formulated as a graph partitioning problem for which we present an algorithm that is guaranteed to find the optimal solution in polynomial time.

First, label all the terminals within a cell by assigning an angular and radial coordinate to each one of them. The radial position is the distance of the terminal to the base station. The angular position is the angular distance of the terminal to a reference terminal. Without loss of generality, the reference terminal can be selected arbitrarily and assigned $0^{\circ}$ as its angular position. Each terminal within the cell is represented by a vertex (node) along a ring. Let the position of the vertex on the ring be determined by the angular position of the terminal regardless of its radial position. We assume that all angular positions are unique. The radius of the ring is independent of the radial position of the users. The segment of the ring that connects each pair of neighboring nodes is called an edge. Fig. 2 demonstrates the relation between the physical location of terminals and the corresponding nodes along a ring.

The topology just described constitutes a ring $G=(V, E)$, with vertices $V=\left\{v_{1}, v_{2}, \ldots, v_{M}\right\}$ and edges $E=\left\{\left(v_{1}, v_{2}\right)\right.$, $\left.\ldots,\left(v_{M-1}, v_{M}\right)\right\} \cup\left\{\left(v_{M}, v_{1}\right)\right\}$. Denote element $\left(v_{i}, v_{j}\right)$ of the edge set by $e_{i}$. Let the weight of vertex $v_{i}$ be denoted by $w_{i}$ and the weight of edge $e_{i}$ be denoted by $c_{i}$. Vertex $i$ is assigned weight $w_{i}=1 / h_{i}$ where $h_{i}$ is the uplink gain of the $i$ th terminal. The weight of edge $i, c_{i}$, is the angular distance between the two vertices connected by this edge.

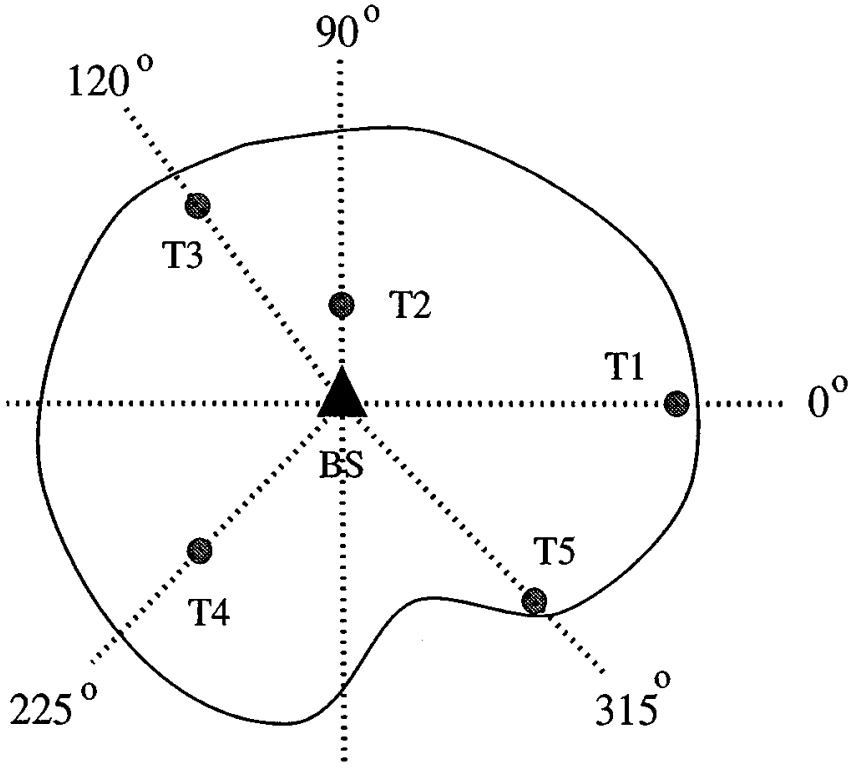

(a)

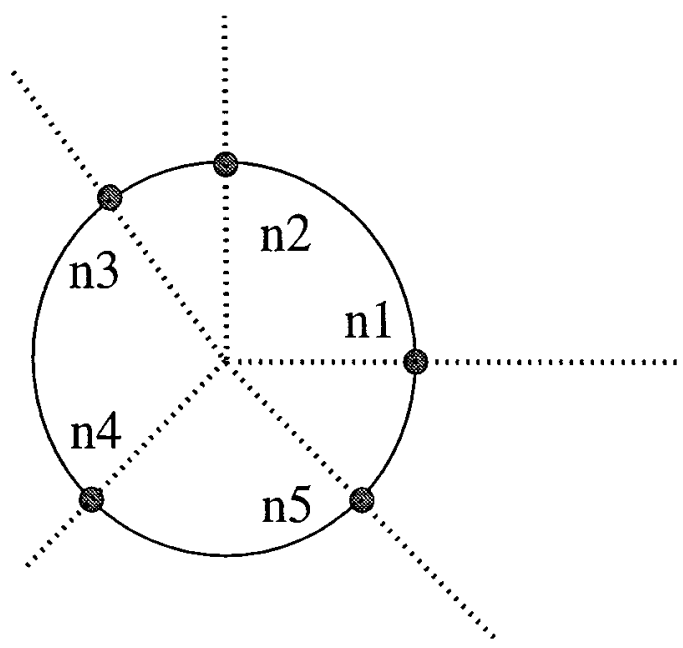

(b)

Fig. 2. (a) Terminal locations within a cell. (b) The graph that is constructed based on the terminal locations. Node $n_{i}$ in graph corresponds to terminal labeled $\mathrm{T} i$ for all $i$.

It is worth noting that two or more randomly placed terminals sharing the same angular location is a probability zero event. Nevertheless, the model is able to handle in case such a degenerate situation actually occurs. If there exist two such terminals, the ring is modified by combining these terminals to form a single vertex and assign a weight that is the summation of the weights of the original nodes. This reduction can be done with no effect on the optimal sectorization outcome since it is physically not possible to place users sharing the same angular position into different sectors.

The sectorization problem for minimum transmitted power (TP) solves for an optimal set of sector angles that identify sector boundaries. The set of terminals in the cell are optimally grouped into disjoint subsets. Graph theoretic methods emerge as powerful tools to find the best partition.

In the next section, we will present general results in graph partitioning. These results apply to optimal partitioning on a string which is a graph where all vertices are located along a 


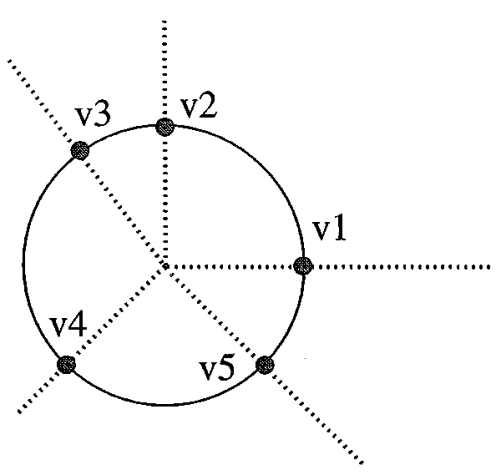

(a)

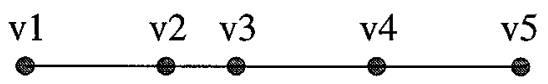

(b)

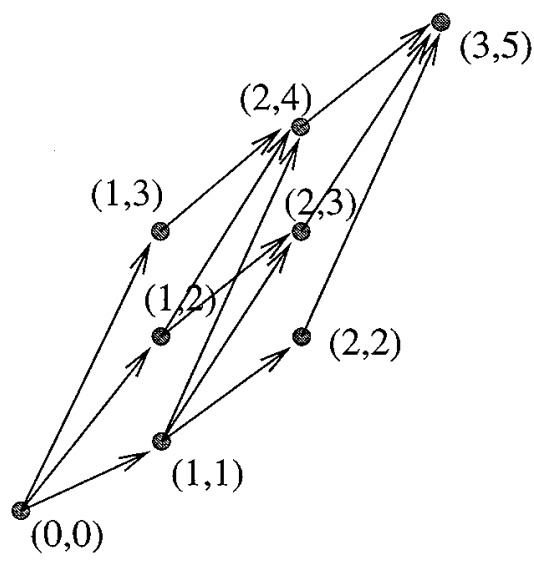

(c)

Fig. 3. (a) Ring that is constructed based on terminal locations within cell. (b) String obtained by removing $e_{5}$. (c) Network constructed from string that is to be partitioned into 3 subsets.

line. An immediate observation is that a ring broken at an edge results in a string as shown in Fig. 3(a) and (b). We will use this fact in Section IV-D.

\section{Optimal Partitioning and Shortest Path Algorithms}

We now digress briefly in order to provide some background for the graph theoretic concepts that are relevant to the setting of the TP problem. Given a graph $G=(V, E)$, let $\pi=$ $\left\{S_{1}, \ldots, S_{N}\right\}$ be a partition of the set of vertices $V$ into $N$ subsets. $\pi$ is considered feasible in $G$ if for every $k \in\{1, \ldots, N\}$, the subgraph $G\left(S_{k}\right)$ induced by $S_{k}$ is connected, and the weight $W\left(S_{k}\right)$ of $S_{k}$ as defined below is greater than or equal to zero. The set of all such feasible partitions is denoted by $\Pi(G, N)$. Note that in a feasible partition, $S_{k} \neq \emptyset$ for all $k$. The cost function $C: \Pi(G, N) \rightarrow \Re$ assigns a value to each feasible partition and the cost of a particular partition $\pi=\left\{S_{1}, \ldots, S_{N}\right\}$ is given by

$$
C(\pi)=\sum_{i=1}^{N} W\left(S_{i}\right)
$$

The graph partitioning problem (GP) is to find a feasible partition such that some given cost is minimized. Mathematically, it can be expressed as

$$
\min _{\pi=\left\{S_{1}, \ldots, S_{N}\right\} \in \Pi(G, N)} C(\pi)=\sum_{i=1}^{N} W\left(S_{i}\right) \quad(\mathbf{G P}) .
$$

We now show that the graph partitioning problem GP is polynomially solvable under certain conditions. We first observe that the cost function of GP given in (13) is separable. This fact follows from the definition of a separable function given below.

Definition 1: A function of $M$ variables $f\left(x_{1}, x_{2}, \ldots, x_{M}\right)$ is said to be separable if it can be expressed as a sum of $M$ functions of a single variable; i.e., $f\left(x_{1}, x_{2}, \ldots, x_{M}\right)=$ $\sum_{i=1}^{M} f_{i}\left(x_{i}\right)$.

Although the problem of optimally partitioning an arbitrary graph with an arbitrary cost function is an NP-hard optimization problem, partitioning a string optimally with a separable cost function can be solved in polynomial time. We use the following optimal graph partitioning result presented in [20].

Theorem 1: If the objective function is separable, the problem of optimally partitioning a string can be reduced to a shortest path problem with computational complexity $\mathcal{O}\left(M^{2} N\right)$.

We provide a sketch of the proof here to present the general idea behind it. A full proof can be found in [20]. The key point is to establish a one-to-one correspondence between the cost of a feasible partition in a string and the cost of a path from the origin to destination in a special acyclic network obtained from the partitioning problem at hand. An acyclic network is a directed graph with a length associated with each edge and that has no paths that start and end at the same node.

Suppose $H$ is a string with $M$ vertices labeled $v_{1}, \ldots, v_{M}$ so that adjacent vertices have consecutive indices and let the weight of the vertices be denoted as $w_{1}, \ldots, w_{M}$. An edge $e_{i}$ exists between vertex $v_{i}$ and $v_{i+1}$ and has weight $c_{i}$. $H$ is to be partitioned into $N$ subsets such that a separable objective function is optimized. Suppose that in any feasible partition $\left\{S_{1}, \ldots, S_{N}\right\}$, adjacent subsets have consecutive indices. Given the string and the problem, construct an acyclic network $Q$ as follows. Let $(0$, $0)$ and $(N, M)$ be the origin and the destination nodes in $Q$, respectively. The nodes that lie between the origin-destination pair are given by the set

$$
\{(k, i): 1 \leq k \leq N-1 ; k \leq i \leq M-N+k\}
$$

An edge is placed from node $(k, i)$ to $(h, j)$ if $h=k+1$ and $j>$ $i$. Otherwise, there is no edge between $(k, i)$, and $(h, j)$. There is a one-to-one correspondence between the feasible partitions of $H$ into $N$ subsets and the paths from $(0,0)$ to $(N, M)$ in $Q$. The path $P$ corresponding to a given $\pi \in \Pi(H, N)$ is defined as follows. The node $(k, i)$ belongs to $P$ if and only if $i$ is the last vertex of the subset $S_{k}$ of $\pi$. Furthermore, when the weight of edge $u$ in $Q$ is assigned the cost of the corresponding subset in $H$, the cost of partition $\pi, C(\pi)$ in (13) coincides with the total length of path $P$ in network $Q$. It follows that the optimum partition of $H$ can be determined by finding a shortest path from 
$(0,0)$ to $(N, M)$ in $Q$ since each path corresponds to one and only one feasible partition.

Fig. 3(b) and (c) shows how the network $Q$ is obtained from the GP problem with $G, M=5$ nodes and $N=3$ partitions. Notice that as a result of the construction of the network, no empty sets are allowed.

A shortest path from origin to destination can be found in $\mathcal{O}\left(M^{2} N\right)$ by using well-known shortest path algorithms. Dijkstra's method-which finds the shortest path from an origin node to the destination node in a network with positive edge lengths_-is particularly suitable for use in our case [21]-[24].

In the next section, we will establish the equivalence of the GP problem and the TP problem and use this fact to present the solution of the TP problem.

\section{Graph Theoretic Solution of TP}

Let us return to the discussion of TP and its solution in the graph theoretic framework. First, we observe that the cost function of TP given in (11) is separable, i.e., the cost of a sectorization arrangement in TP can be expressed as a sum of the costs of the individual sectors. Let $S_{i} \subset V$ represent the set of users that is in the $i$ th sector in a given partition. We define the cost of sector $S_{i}$ by

$$
\begin{aligned}
W\left(S_{i}\right) & =\frac{\gamma^{*} \sigma^{2}}{1-\frac{\gamma^{*}\left(\left|S_{i}\right|-1\right)}{G}} \sum_{k: v_{k} \in S_{i}} w_{k} \\
& =\frac{\gamma^{*} \sigma^{2}}{1-\frac{\gamma^{*}\left(\left|S_{i}\right|-1\right)}{G}} \sum_{k: v_{k} \in S_{i}} \frac{1}{h_{k}}
\end{aligned}
$$

where $\left|S_{i}\right|$ is the number of vertices in $S_{i}$. Then, the total cost, which is the summation of $W\left(S_{i}\right)$ over all sectors, has the same structure as the cost function (13) for GP. In addition, each feasible partition $\pi$ corresponds to a set of cell sectorization configurations $\left\{s_{i}(\boldsymbol{\theta})\right\}$, both of which have the same cost value. Therefore, it follows that a solution for GP is also a solution for TP. It should be noted that the constraints of the TP problem in (11) are accommodated while generating the set of feasible partitions, $\Pi$, for the GP problem in (13). Specifically, suppose that in some partition, for sector $i$, we have $1-\left(\gamma^{*}\left(\left|S_{i}\right|-1\right) / G\right) \leq 0$. In that case, we remove from the set of all possible partitions which have sector $i$ as one of the sectors of the partition. Therefore, an infeasible partition could never emerge as a solution of GP. The TP problem also imposes a constraint through $\boldsymbol{\theta}$, which is mapped to GP as connected partitions. A partition that is not connected is considered infeasible while constructing $\Pi$.

Notice that the graph theoretic formulation returns the optimal sectors in terms of a list of terminals that belong to each sector. However, it does not say anything about where the actual sector boundary is located. The choice of the actual sector boundary is flexible since it can lie anywhere between the closest two terminals that belong to different sectors. The location of the boundary between neighboring vertices might have practical significance when considering physical constraints as explained in Section IV-E, however, it has no impact on the cost function and the optimization given in (13).

So far, we established that TP is actually equivalent to GP with a separable objective function. Recall from Theorem 1 that GP with a separable objective function can be reduced to a shortest path problem on a string. We observe that the graph corresponding to TP is a ring instead of the string that Theorem 1 applies for. However, as mentioned in Section IV-B, if the ring in TP is broken at an edge (by removing the edge), the resulting graph is a string. Thus, with some increase in computational complexity, we can solve TP by solving a number of shortest path problems. Recall that the computational complexity of solving for the best connected $N$ partition is $\mathcal{O}\left(M^{2} N\right)$. Repeating the same procedure for each edge in $E$, and choosing the best among these solutions, we end up with the optimal solution for TP which has complexity $\mathcal{O}\left(M^{3} N\right)$. The sectors are then formed based on this optimal partition.

\section{E. Physical Constraints}

In this subsection, we discuss how to incorporate certain physical constraints into the original TP problem. These physical constraints can be accommodated easily in the graph theoretical formulation of TP, and do not bring any additional computational complexity to the solution algorithm. They do, however, have practical significance from a system point of view which is why we address them here. The weight $c_{i}$ for edge $e_{i}$ was defined earlier in Section IV-B as the angular distance between the two vertices connected by $e_{i}$. However, for the results presented up to here, the edge weights had no impact on the optimum solution of the problem. In what follows, physical constraints we consider do appear in the form of constraints on the edge weights and thus affect the optimum solution.

The three constraints we consider are 1) minimum allowable angular distance between two users that belong to two neighboring sectors, 2) maximum allowable sector width, and 3) minimum allowable sector width. The first constraint is needed to ensure that two users that are physically very close to each other are not assigned to different sectors, since accomplishing such precise beamforming could be difficult. We modify the vertex set such that vertices that are "very close" to each other are combined to form a supernode. This ensures that two such nodes are not placed in different sets in a partition. The minimum angular distance between two users in neighboring sectors, $c_{\text {min }}$, is a system design parameter. The edges with weight, $c_{i}<c_{\text {min }}$, are deleted from the edge set, and the two neighboring nodes are combined to form a supernode which is placed midway along the edge that is deleted. The weight of the supernode and the edges that connect it to the rest of the ring are adjusted appropriately.

Next, let us turn our attention to the next two constraintsmaximum and minimum sector size. Suppose the maximum and minimum allowable sector width is denoted by $\theta_{\max }$ and $\theta_{\min }$, respectively. We require that $\theta_{\min }<\theta_{i}<\theta_{\max }$ for all sectors $i$. This constraint can be incorporated in the solution described in Section IV-C by modifying the way edges are assigned in the network. An infinite weight is assigned to edges in the network that correspond to sectors that are wider or narrower than the specified limit. Thus, while searching for a shortest path, such sectors do not emerge as possible solutions. The rest of the procedure of finding the optimal partition remains the same.

Recall that without constraints, the optimal sector boundaries are flexible, i.e., as long as each sector contains the optimal set of users, the exact locations of sector boundaries are not im- 
portant. However, with constraints present, the angular space between neighboring terminals becomes significant, and hence boundaries less flexible. It is also worth noting that feasibility issues arise with sector size constraints. It may be the case that there exists no sectorization configuration satisfying a particular size constraint. For example, it is not possible to find three sectors each less than $120^{\circ}$.

\section{Multicell EnVironment}

In this section, optimal cell sectorization and transmit power assignment for a cell in a multicell system is considered. The scenario we consider is appropriate for enhancing the capacity of a highly loaded cell, a hot spot, in the network. It is assumed that the transmit power values for out-of-cell interferers are assigned and do not change significantly as a result of the sectorization of the hot spot cell. Suppose a cell with $M$ users is to be divided into $N$ sectors where $L$ users interfere from outside the cell. Define the out-of-cell interference (OCI) profile, $I(\phi)$, as a discrete function that gives the value of the interference power seen at the base station of the cell to be optimally sectorized. It is a function of $\phi$, the direction of interference; i.e., the value of the OCI function at $\phi_{k}$ is denoted by $I_{k}=I\left(\phi_{k}\right)$ and is the contribution of the $k$ th out of cell interferer which has angular distance $\phi_{k}$ to the cell to be optimally sectorized. Thus, the OCI function has nonzero value only at a set of angle values $\phi_{k}$ where $k=1, \ldots, L$. (Fig. 7 shows a sample OCI profile.) We will assume that $I(\phi)$ is known at the base station of the hot spot cell.

The level of OCI of any given user in the hot spot cell depends on the area covered by its sector. In particular, the OCI that user $j \in s_{i}$ experiences in sector $i$ which covers a region from $\alpha^{i-1}=\sum_{j=1}^{i-1} \theta_{j}$ to $\alpha^{i}=\sum_{j=1}^{i} \theta_{j}$ can be expressed as

$$
I^{i}=\sum_{\left\{k \mid \alpha^{i-1} \leq \phi_{k} \leq \alpha^{i}\right\}} I_{k} .
$$

Recall that $a_{j}$ denotes the index of the sector that user $j$ belongs to. For any two users $j, k \in s_{i}$ in the same sector, the OCI are $I^{a_{j}}=I^{a_{k}}=I^{i}$.

Note that, since the OCI is a function of the sector width, optimal cell boundaries are no longer flexible as is the case in the single cell scenario. To limit the infinite degree of freedom in selection of sector boundary locations, we constrain the sector boundaries to lie only midway between any pair of neighboring terminals. This limitation will render the optimization problem manageable.

We denote by MTP the multicell transmit power optimization problem. In the presence of OCI, MTP can be formulated as

$$
\begin{array}{ll}
\min _{\boldsymbol{\theta}, \mathbf{p}} & \sum_{i=1}^{N} \sum_{j \in s_{i}(\boldsymbol{\theta})} p_{j} \quad(\mathbf{M T P}) \\
\text { s.t. } & \gamma_{j}=\frac{p_{j} h_{j}}{\sum_{k \neq j} \delta_{k j} p_{k} h_{k} / G+I^{a_{j}}+\sigma^{2}} \geq \gamma^{*} \\
& j=1, \ldots, M \\
& \mathbf{p} \geq \mathbf{0} \quad \mathbf{1}^{T} \boldsymbol{\theta}=2 \pi .
\end{array}
$$

Notice that the OCI contributes the additional interference term in the denominator of the SIR in the above equation. Once again, observe that at the optimum solution of MTP, $\gamma_{j}=\gamma^{*}$ for all users and users belonging to the same sector should have equal received power at the base station. Then, (17) can be rewritten as,

$$
\begin{aligned}
& \min _{\boldsymbol{\theta}, M_{i}} \sum_{i=1}^{N} \frac{\gamma^{*}\left(\sigma^{2}+I^{i}\right)}{1-\frac{\gamma^{*}\left(M_{i}(\boldsymbol{\theta})-1\right)}{G}} \sum_{j \in s_{i}(\boldsymbol{\theta})} \frac{1}{h_{j}} \\
& M_{i}(\boldsymbol{\theta})<G / \gamma^{*}+1 \quad \sum_{i=1}^{N} M_{i}(\boldsymbol{\theta})=M \quad \mathbf{1}^{T} \boldsymbol{\theta}=2 \pi .
\end{aligned}
$$

A comparison between (11) and (18) reveals the difference between the optimization problem in a single cell and a multicell scenario. With OCI present, the total interference experienced in sector $i$ is $\sigma^{2}+I^{i}$ as opposed to the AWGN power of $\sigma^{2}$ when there is no out-of-cell interference. The alternative expression of the MTP in (18) allows us to observe that the formulation of the problem as a graph partitioning problem is still possible with slight changes to the methodology already explained in Section IV. In particular, the weight of a subset $S$ of the set of vertices $V$ should be modified as follows:

$$
W(S)=\frac{\gamma^{*}\left(\sigma^{2}+I^{S}\right)}{1-\frac{\gamma^{*}(|S|-1)}{G}} \sum_{k: v_{k} \in S} w_{k}
$$

where $w_{k}=1 / h_{k}$ and $I^{S}$ is the OCI associated with subset $S$. It remains to calculate $I^{S}$ for each subset $S$ in any given feasible partition. This calculation from (16) brings an additional computational cost to the GP problem, but the complexity of the problem remains polynomial.

We can also formulate a multicell received power optimization problem (MRP) similar to the MTP formulation above as

$$
\begin{array}{ll}
\min _{\boldsymbol{\theta}, \mathbf{p}} & \sum_{i=1}^{N} \sum_{j \in s_{i}(\boldsymbol{\theta})} p_{j} h_{j} \quad(\mathbf{M R P}) \\
\text { s.t. } & \gamma_{j}=\frac{p_{j} h_{j}}{\sum_{k \neq j} \delta_{k j} p_{k} h_{k} / G+I^{a_{j}}+\sigma^{2}} \geq \gamma^{*} \\
& j=1, \ldots, M \quad \mathbf{p} \geq \mathbf{0} \quad \mathbf{1}^{T} \boldsymbol{\theta}=2 \pi .
\end{array}
$$

As in Section III, we observe that the SIR constraints are met with equality and that the received powers of any two terminals within the same sector are equal at the optimal allocation. As a result, MRP in (20) can be simplified to give

$$
\begin{aligned}
\min _{\boldsymbol{\theta}, M_{i}} & \sum_{i=1}^{N} \frac{M_{i}(\boldsymbol{\theta}) \gamma^{*}\left(\sigma^{2}+I^{i}\right)}{1-\frac{\gamma^{*}\left(M_{i}(\boldsymbol{\theta})-1\right)}{G} \quad(\text { MRP })} \\
& M_{i}(\boldsymbol{\theta})<G / \gamma^{*}+1 \quad \sum_{i=1}^{N} M_{i}(\boldsymbol{\theta})=M \quad \mathbf{1}^{T} \boldsymbol{\theta}=2 \pi .
\end{aligned}
$$

Due to the OCI term in the cost function, a simple solution similar to the closed form solution of Section III is not available in the multicell setting. However, we can use the graph theoretic tools explained in this paper to solve the MRP optimally. More specifically, when constructing the network corresponding to 


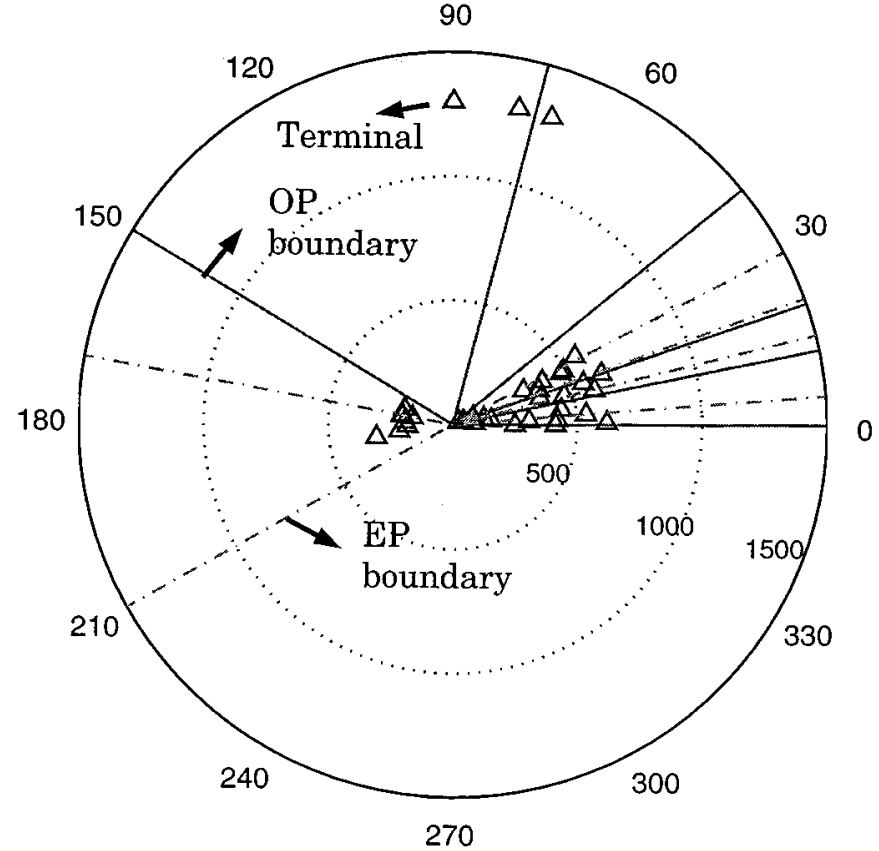

Fig. 4. Sector boundaries of $\mathrm{OP}$ and $\mathrm{EP}$ in a cell where the number of terminals $M=36$, number of sectors $N=6$, processing gain $G=64$, target SIR $\gamma^{*}=7 \mathrm{~dB}$, and noise power $\sigma^{2}=10^{-13}$.

the optimization problem, the weight of a subset $S$ of the set of vertices $V$ should be assigned as

$$
W(S)=\frac{|S| \gamma^{*}\left(\sigma^{2}+I^{S}\right)}{1-\frac{\gamma^{*}(|S|-1)}{G}}
$$

where $I^{S}$ is the OCI associated with subset $S$. Once these weights are obtained, the solution method is identical to MTP.

In the next section, we will provide examples both for the single cell system and for a hot spot cell in a multicell setting, and compare the results of several different sectorization methods.

\section{NUMERICAL RESULTS}

We provide sectorization results by three methods: transmit power optimization, received power optimization, and uniform sectorization. The output of the algorithm given in Section IV-C is the optimal partitioning $(\mathrm{OP})$ that minimizes sum of transmit powers of all users in the cell. Sectorization that places an equal number of terminals in each sector, equal population sectorization (EP), is the minimum received power result. Finally, in uniform sectorization (UP), sectors are of equal width and their orientation does not depend on the terminal locations.

First, we consider the single cell system. Fig. 4 shows sector boundaries obtained by OP and EP. For this particular example, UP is not feasible, i.e., the system cannot support acceptable SIR to all users in the cell if uniform sectorization of the cell is employed. Adaptive sectorization enables the system to support all users. For this example, we observe that this is accomplished by placing narrower sectors where users are more clustered and isolating users with large path losses, which is intuitively pleasing. Recall that $\mathrm{OP}$ has complexity $\mathcal{O}\left(M^{3} N\right)$. Since $\mathrm{EP}$ is much easier to implement, one might wonder how EP performs in terms of total transmit power. We have compared the
TABLE I

Total Transmit Powers [WATTS] OBTAINEd From THREe MethodS

\begin{tabular}{l|c|c}
\hline & Figure 4 & Figure 5 \\
\hline TTP(OP) & 6.3 & 3.9 \\
\hline TTP(EP) & 9.1 & 4.2 \\
\hline TTP(UP) & Infeasible & 5.8 \\
\hline
\end{tabular}

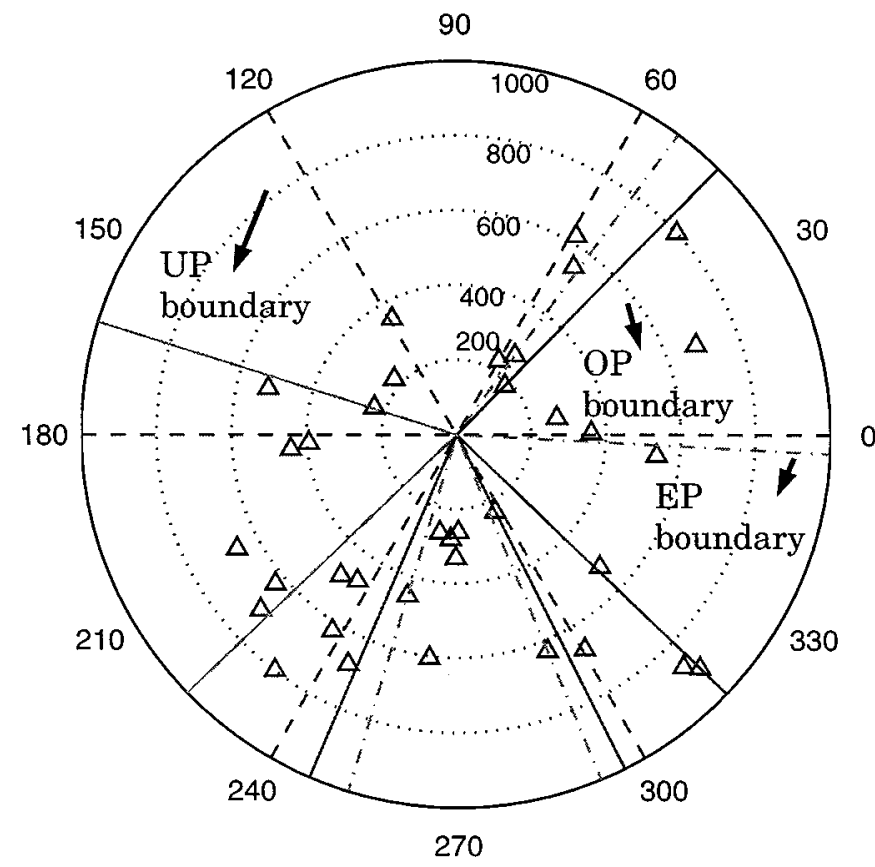

Fig. 5. Sector boundaries of OP, EP, and UP in a cell where the number of terminals $M=36$, number of sectors $N=6$, processing gain $G=64$, target $\operatorname{SIR} \gamma^{*}=7 \mathrm{~dB}$, and noise power $\sigma^{2}=10^{-13}$. The distribution of terminals is uniform in area.

total transmit power values for all three methods in Table I. It can be seen that, for this example, OP and EP result in quite different total transmit power values, and OP brings $40 \%$ improvement in total transmit power (TTP) as compared to EP. This is an example where finding OP really pays off in terms of transmit power savings.

Next, we consider a scenario where the distribution of the terminals are uniform in area within the cell. The resulting sectorization arrangements are shown in Fig. 5. For this example, the total transmit power of OP [TTP(OP)] is about $32 \%$ less than the total transmit power of UP [TTP(UP)]. However, OP's improvement over EP is only 7\% (Table I). In general, in case of uniformly distributed terminals, TTPs with OP, EP, and UP are expected to be close to each other. An extreme case where all three methods yield the same TTP is when the terminals lie along the perimeter of a circle at uniform angular spacing.

The effect of the sector width constraint on the optimum partitioning is displayed in Fig. 6. In this example, the OP with no constraints yields a transmit power of $6.3 \mathrm{~W}$. When the sector width is constrained to $120^{\circ}$, the OP results in $6.6 \mathrm{~W}$, and the sector boundaries are altered as shown in the figure. The two other constraints we discussed earlier can also be easily accommodated with no increase in computational complexity.

In the multicell scenario, we study sectorization of a center cell that is surrounded by interfering cells on a 9-cell grid. 


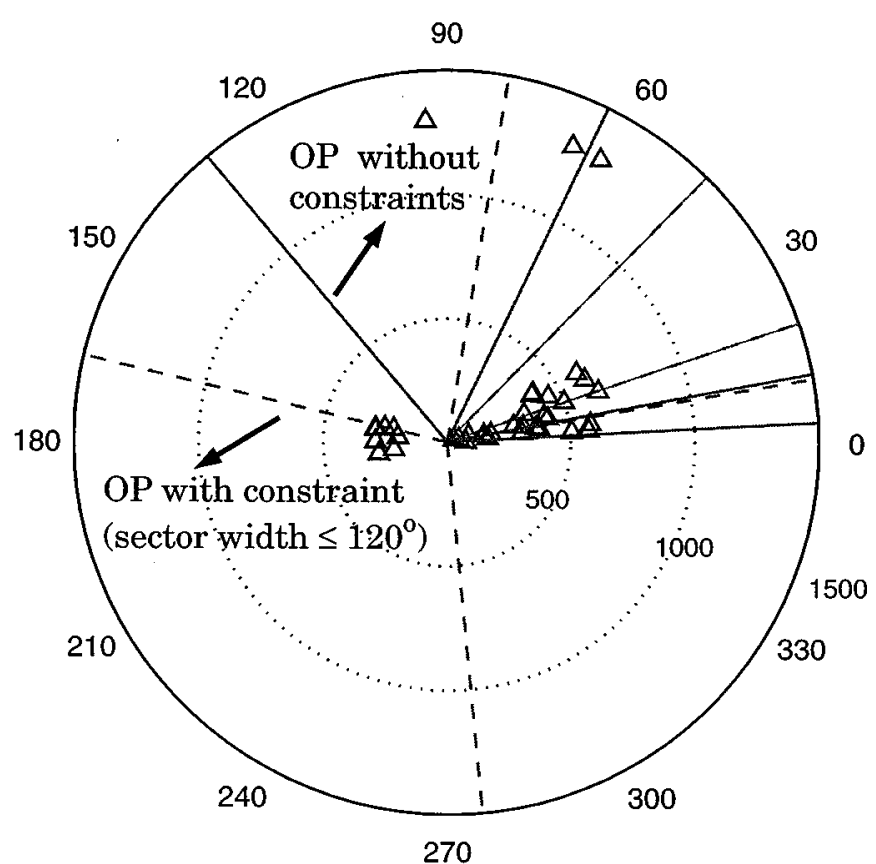

Fig. 6. Sector boundaries of OP, both without any constraints and with sector width constrained to $120^{\circ}$, where the number of terminals $M=36$, number of sectors $N=6$, processing gain $G=64$, target $\operatorname{SIR} \gamma^{*}=7 \mathrm{~dB}$, and noise power $\sigma^{2}=10^{-13}$.

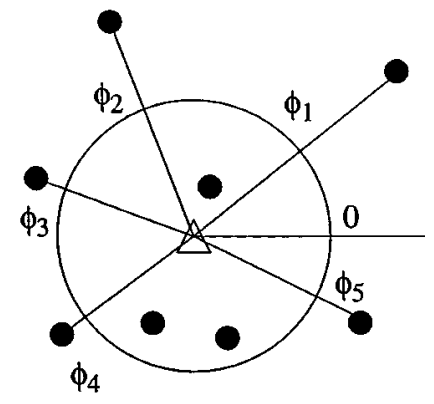

(a)

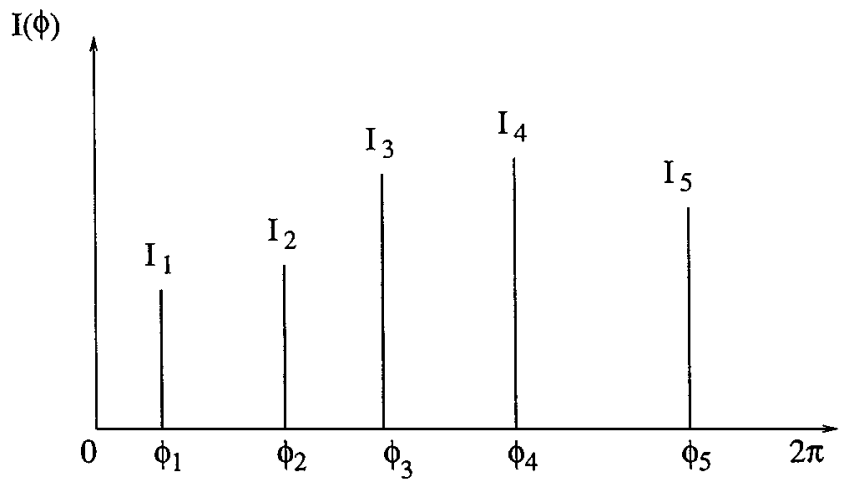

(b)

Fig. 7. (a) Terminals outside the coverage area of the cell cause interference at the base station at the given angular positions. (b) The level of interference caused by each user maps to an interference profile where interference power at the base station is plotted as a function of the direction of interference.

We examine the worst-case scenario by assigning maximum transmit power to all the out-of-cell interferers. Maximum transmit power level is taken to be $1 \mathrm{~W}$. We compare both uniform sectorization (UP) and equal population sectorization (EP) with optimal sectorization (OP) obtained by solving the

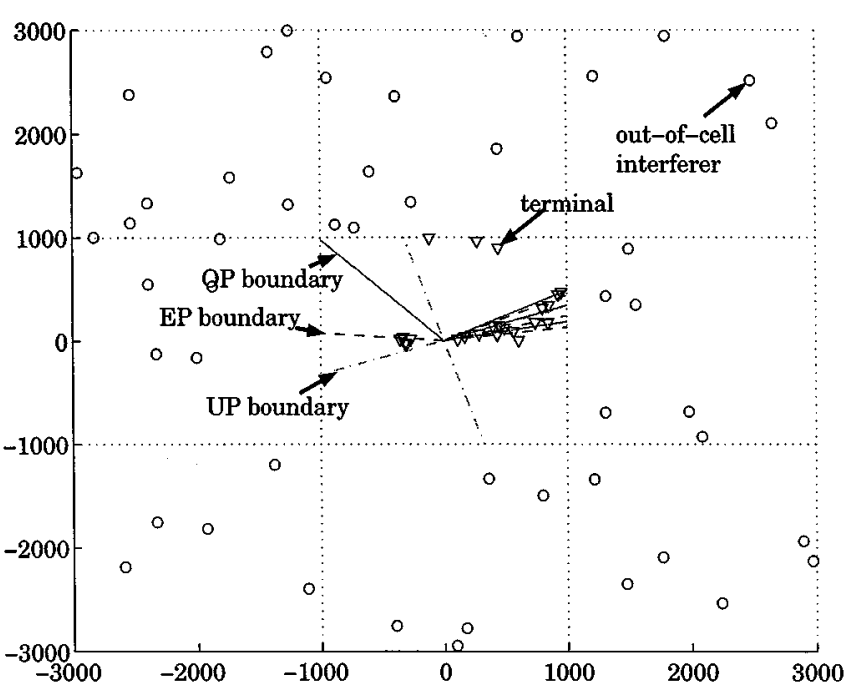

Fig. 8. Sector boundaries of OP, EP, and UP in a cell with no OCI where the number of terminals $M=24$, number of sectors $N=4$, processing gain $G=64$, target SIR $\gamma^{*}=7 \mathrm{~dB}$, and noise power $\sigma^{2}=10^{-13}$. The number of out-of-cell interferers is $L=48$ and they are distributed uniformly, but they do not transmit in this case.

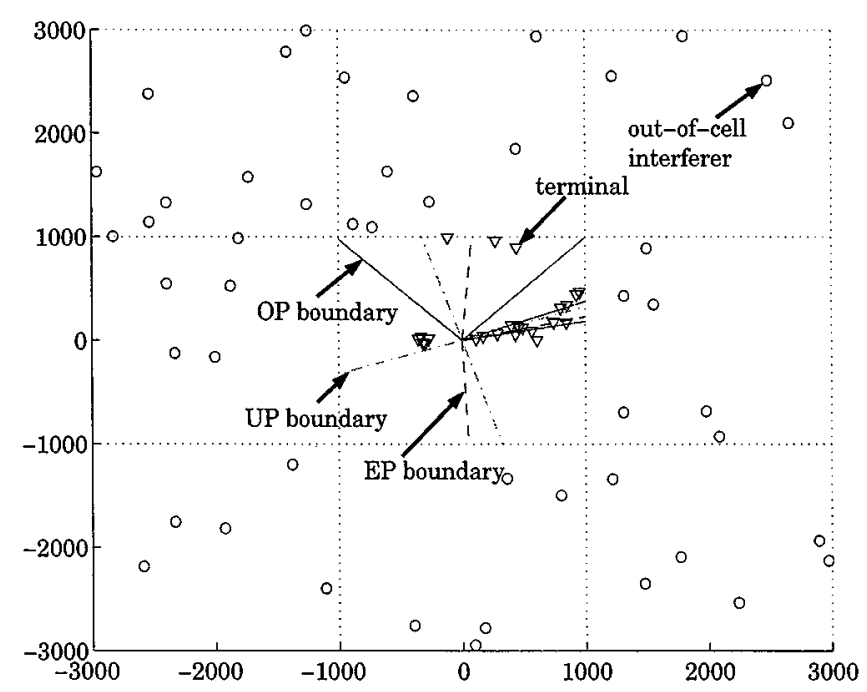

Fig. 9. Sector boundaries of OP, EP, and UP in a cell with OCI where the number of terminals $M=24$, number of out-of-cell interferers $L=48$, number of sectors $N=4$, processing gain $G=64$, target $\operatorname{SIR} \gamma^{*}=7 \mathrm{~dB}$, and noise power $\sigma^{2}=10^{-13}$. The out-of-cell terminals transmit with maximum power of $1 \mathrm{~W}$. The distribution of out-of-cell terminals is uniform. Same setting as in previous figure.

MTP problem explained in Section V. UP is obtained by selecting the best partition, i.e., the partition that yields the lowest total transmit power, among all the partitions where each sector is the same size. EP is obtained by selecting the best partition among all partitions that place an equal number of terminals in each sector. OP boundaries represent the solution of the MTP obtained by using the graph theoretic transformation explained earlier. For computational ease, we assume that a boundary can be placed only halfway between any two terminals as opposed to the infinitely many possible boundary placement. Fig. 8 shows sector boundaries for uniform sectorization (UP), equal population sectorization (EP), and optimal sectorization (OP) when no out-of-cell interferers are active. Fig. 9 shows UP, EP, and OP boundaries for the same system when all out-of-cell 
TABLE II

TOTAL TRANSMIT POWERS [WATTS] OBTAINED FROM OP, EP, AND UP IN THE MULTICELL SCENARIO

\begin{tabular}{l|c|c}
\hline & Figure 8 (no OCI) & Figure 9 (with OCI) \\
\hline TTP(OP) & 6.6 & 7.0 \\
\hline TTP(EP) & 7.6 & 8.0 \\
\hline TTP(UP) & 13 & 15 \\
\hline \% improvement & 15,109 & 15,120 \\
\hline
\end{tabular}

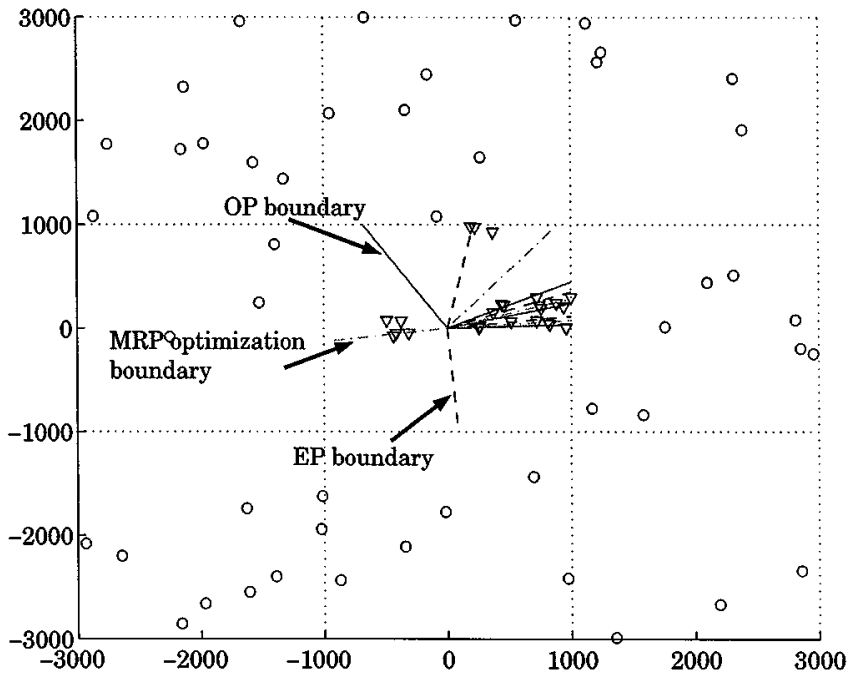

Fig. 10. Comparison between OP boundaries that solve MTP, EP boundaries, and MRP solution boundaries.

terminals transmit at maximum power. Total transmit power (TTP) values are compared in Table II. It is observed that although the total transmit powers increase in the presence of OCI, the result obtained by OP is still substantially better than that provided by UP or EP. OP is clearly superior to the conventional method of UP.

Finally, in Fig. 10 we compare partitions from OP, EP, and the partitions from the solution of the multicell received power optimization problem (MRP) introduced in Section V. It is interesting to note that the MRP solution yields a partition that places the same number of terminals in each sector, but is different from the EP solution. Furthermore, in terms of transmit powers, MRP yields a higher TTP than both OP and EP. Note that in the absence of OCI power, the EP solution and the MRP solution are exactly the same.

\section{CONCLUSION}

In this paper, we studied the joint total power optimization and adaptive sectorization problem for both the received and transmit power optimization cases. It is shown that the total received power is minimized by equalizing the number of users in each sector (EP), while the transmit power optimizing partition (OP) can be found with polynomial complexity using a graph theoretical approach. OP is expected to reduce total transmit power the most in scenarios similar to the one given in Fig. 4 where terminal locations are nonuniform in the cell. We also studied sectorization of a hot spot cell in the presence of out-of-cell interference. We used the same graph theoretic tools as in the previous case with slight modifications to the formulation under the assumption that the interference profile is available at the base station of the cell in question. Results obtained in both the single cell and the multicell scenarios show transmit power savings and strongly encourage the use of adaptive sectorization to support more users for a given SIR target.

The graph theoretical approach introduced in this paper is fairly general and can accommodate several different constraints that are encountered in practical systems. To this end, we have shown the accommodation of, and provided results for, physical constraints related to the sizes and the formation of different sectors. We note that it is possible to accommodate maximum and minimum transmit power constraints into this formulation as well. The existence of these extra constraints is reflected in the construction of the feasible partitions $\Pi$, i.e., a partition where the maximum or minimum power constraint violated simply is not included in $\Pi$.

Finally, the motivation behind the present paper is to enhance the uplink capacity of CDMA systems. Equally interesting issues arise and are currently being investigated when downlink is considered to be the capacity limiting link, as may be the case for future wireless systems with internet browsing capability.

\section{ACKNOWLEDGMENT}

The authors thank Dr. B. Simeone and Dr. T. Ünlüyurt for helpful discussions throughout this work. They also thank Dr. R. Yates for helpful comments on the manuscript.

\section{REFERENCES}

[1] A. J. Viterbi, CDMA: Principles of Spread Spectrum Communication. Reading, MA: Addison-Wesley, 1995.

[2] J. Zander, "Performance of optimum transmitter power control in cellular radio systems," IEEE Trans. Veh. Technol., vol. 41, pp. 57-62, Feb. 1992.

[3] S. Grandhi, R. Vijayan, D. J. Goodman, and J. Zander, "Centralized power control in cellular radio systems," IEEE Trans. Veh. Technol., vol. 42, pp. 466-468, Nov. 1993.

[4] J. Zander, "Distributed cochannel interference control in cellular radio systems," IEEE Trans. Veh. Technol., vol. 41, pp. 305-311, Aug. 1992.

[5] G. J. Foschini and Z. Miljanic, "A simple distributed autonomous power control algorithm and its convergence," IEEE Trans. Veh. Technol., vol. 42, pp. 641-646, Nov. 1993.

[6] R. D. Yates, "A framework for uplink power control in cellular radio systems," IEEE J. Select. Areas Commun., vol. 13, pp. 1341-1347, 1995.

[7] R. D. Yates and C. Y. Huang, "Integrated power control and base station assignment," IEEE Trans. Veh. Technol., vol. 44, pp. 638-644, Aug. 1995.

[8] S. V. Hanly, "An algorithm of combined cell-site selection and power control to maximize cellular spread spectrum capacity," IEEE J. Select. Areas Commun., vol. 13, pp. 1332-1340, Sept. 1995.

[9] S. Ulukus and R. D. Yates, "Adaptive power control and MMSE interference suppression," ACM Wireless Networks, Special Issue on Multiuser Detection in Wireless Communications, vol. 4, no. 6, pp. 489-496, Nov. 1998.

[10] F. Rashid-Farrokhi, L. Tassiulas, and K. J. R. Liu, "Joint power control and beamforming in wireless networks using antenna arrays," IEEE Trans. Commun., vol. 46, pp. 247-256, 1998.

[11] A. Yener, R. D. Yates, and S. Ulukus, "Joint power control, multiuser detection and beamforming for CDMA systems," in Proc. IEEE VTC, May 1999 , pp. 1032-1036.

[12] D. N. C. Tse and S. V. Hanly, "Linear multiuser receivers: Effective interference, effective bandwidth and user capacity," IEEE Trans. Inform. Theory, vol. 45, pp. 641-657, Mar. 1999. 
[13] Y. Guo and B. Aazhang, "Capacity of multi-class traffic CDMA system with multiuser receiver," in Proc. IEEE WCNC, 1999, pp. 500-504.

[14] B. D. Van Veen and K. M. Buckley, "Beamforming: A versatile approach to spatial filtering," IEEE Acoust., Speech, Signal Processing Mag., pp. 4-24, Apr. 1988.

[15] A. J. Paulraj and C. B. Papadias, "Space-time processing for wireless communications," IEEE Signal Processing Mag., pp. 49-83, Nov. 1997.

[16] G. K. Chan, "Effects of sectorization on the spectrum efficiency of cellular radio systems," IEEE Trans. Veh. Technol., vol. 41, pp. 217-225, Aug. 1992.

[17] V. K. Garg and E. L. Sneed, "Digital wireless local loop system," IEEE Commun. Mag., pp. 112-115, Oct. 1996.

[18] M. R. Garey and D. S. Johnson, Computers and Intractability. San Francisco, CA: W.H. Freeman, 1979.

[19] B. Simeone. (1999, May) Optimal connected partitions on graphs. Tutorial Series at DIMACS Center, Rutgers Univ., NJ. [Online]. Available: http://rutcor.rutgers.edu/ boros/LSDO/BrunoSimeone.html

[20] C. De Simone, M. Lucertini, S. Pallottino, and B. Simeone, "Fair dissections of spiders, worms, and caterpillars," Networks, vol. 20, pp. 323-344, 1990

[21] E. L. Lawler, Combinatorial Optimization: Networks and $\mathrm{Ma}$ troids. New York: Holt, Rinehart, Winston, 1976.

[22] T. Lengauer, Combinatorial Algorithms for Integrated Circuit Layout. New York: Wiley, 1990.

[23] D. P. Bertsekas and R. Gallager, Data Networks, 2nd ed. Englewood Cliffs, NJ: Prentice-Hall, 1992.

[24] M. S. Bazaraa, J. J. Jarvis, and H. D. Sherali, Linear Programming and Network Flows, 2nd ed. New York: Wiley, 1990.

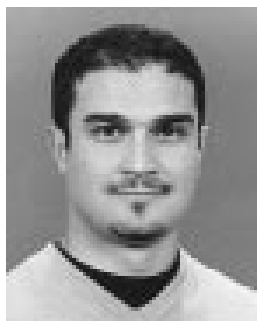

Cem U. Saraydar was born in Silifke, Turkey, in 1971. He received the B.S. degree in 1993 from College of Engineering, Bogazici University, Istanbul; and the M.S. and Ph.D. degrees in electrical engineering from Rutgers University, New Brunswick, NJ, in 1997 and 200, respectively. During his Ph.D. studies, he was with the Wireless Information Network Laboratory (WINLAB), Department of Electrical and Computer Engineering, Rutgers University.

Since October 2000, he has been with Bell Labs, Lucent Technologies. His current research interests include optimal pricing in wireless data networks, applications of game theory in wireless networks, graph theoretic models in communications systems, and mobility management in cellular systems.

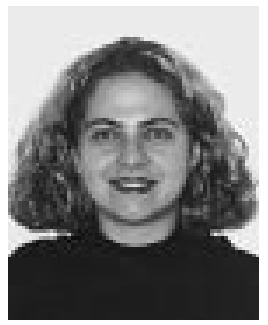

Aylin Yener received the B.S. degree in electrical and electronics engineering and in physics, both from Bogazici University, Istanbul, Turkey; and the M.S. and Ph.D. degrees in electrical and computer engineering from Rutgers University, NJ, in 1991, 1991, 1994, and 2000, respectively.

During her Ph.D. studies, she was with the Wireless Information Network Laboratory (WINLAB) in the Department of Electrical and Computer Engineering at Rutgers University. Since 2000, she has been with the Department of Electrical Engineering and Computer Science at Lehigh University, PA, as an Assistant Professor. Her research interests include performance enhancement for CDMA systems, wireless communication theory, and wireless networking in general. 